\title{
Intelligent Video Surveillance Technology in Intelligent Transportation
}

\author{
Fangcheng He \\ School of Foreign Languages, Chongqing University of Education, Chongqing, China \\ Correspondence should be addressed to Fangcheng He; gxwpc@cque.edu.cn
}

Received 26 March 2020; Revised 29 July 2020; Accepted 3 September 2020; Published 16 November 2020

Academic Editor: Wenqing Wu

Copyright (c) 2020 Fangcheng He. This is an open access article distributed under the Creative Commons Attribution License, which permits unrestricted use, distribution, and reproduction in any medium, provided the original work is properly cited.

\begin{abstract}
Along with the strength of the country's overall strength, the people's pockets have become more and more popular, and there have been significant improvements in all aspects of life, especially in terms of travel methods. This reflects the increase in residents' income, but it also brings huge traffic pressure. In the long run, traffic congestion is not only detrimental to urban development, but frequent traffic accidents threaten residents' travel safety. Effective monitoring methods are essential to solving these problems, so it is necessary to carry out research on intelligent video monitoring technology in intelligent transportation. The purpose of this article is to solve the current situation of excessive traffic pressure in the city. Through the study of intelligent video surveillance technology in intelligent traffic, the use of constrained least squares algorithm to remove motion blur and apply Kalan filtering to the sharpening process is used to eliminate noise ambiguity and make a brief introduction to various classic moving target detection methods to realize real-time monitoring of intelligent traffic conditions and continuously adjust and verify the monitoring situation, and then establish intelligent video in intelligent traffic monitoring technology research system. The research results show that this kind of intelligent video surveillance technology research in intelligent transportation can effectively increase the awareness of intelligent video surveillance technology and improve the level of intelligent video surveillance technology. The data measurement time has been shortened by one hour, the aggregation time has been changed from three hours to two hours, and the analysis time has been shortened by half. Eased urban traffic road pressure and greatly reduced the incidence of traffic accidents, which is conducive to socialist harmony social construction.
\end{abstract}

\section{Introduction}

Since ancient times, transportation has been the basic industry of the national economy, an important factor determining national economic development and a basic condition for social development and the improvement of people's living standards $[1,2]$. Since the founding of the People's Republic of China, China has realized a great transition from standing up to becoming rich to becoming strong [3]. With the overall strength of the country, people's pockets are getting bigger and bigger, and they have improved significantly in all aspects of life, especially in the way they travel. From bicycles in the 1980s and 1990s to today's buses, subways, and private cars, both in number and type, have undergone qualitative changes [4-6]. But at the same time, the rapid increase in the number of vehicles has brought great pressure to urban traffic roads. This is not only reflected in the poor effect of coordinating reasonable road flow but also reflects a large amount of material resources and manpower spent in maintaining road order. More distressing is the rapid rise in the incidence of traffic accidents caused by too many vehicles $[7,8]$. These pressures pose a huge test to urban traffic, restrict the efficiency of various urban development work, and bring safety risks to residents' safe travel [9]. In the face of more and more severe traffic pressure, the traditional method has been unable to deal with a good deal. Some cities have adopted the method of limiting the number of orders to solve traffic problems. In fact, this method is essentially to reduce the number of traveling vehicles, thus reducing the traffic pressure [10]. But after all, this method is at the expense of some people's interests and cannot be used for a long time. Another 
phenomenon is the widespread construction of roads to accommodate the increasing number of vehicles. But its problem is also obvious: road design is often not reasonable enough to harm the long-term development of the city [11]. Even more unrealistic is the hope that civilized traffic awareness propaganda will change this situation [12]. Admittedly, the moral quality of Chinese residents has been improved obviously, but this kind of spiritual propaganda is too individual and has little effect. So, we still need a solution to adapt to advanced traffic to solve the huge traffic pressure.

The predecessor of intelligent video surveillance technology research is the intelligent vehicle road system. Intelligent transportation systems effectively integrate advanced information technology, data communication technology, sensor technology, electronic control technology, and computer technology into the entire transportation management system [13]. As a result, a comprehensive, realtime, accurate, and efficient comprehensive transportation and management system has been established that functions in a wide range and in all directions [14]. An intelligent transportation system is a complex and comprehensive system. From the perspective of system composition, it can be divided into the following subsystems: advanced traffic management system, advanced traffic information service system, advanced vehicle control system, freight management system, electronic toll collection system [15], and emergency rescue system [16]. In the expected design of the intelligent traffic detection system, it can greatly improve the efficiency of infrastructure use, thereby reducing government investment in infrastructure facilities; it can also effectively alleviate urban congestion and reduce the incidence of traffic accidents; it is also applied to solve the vehicle exhaust emissions and large vehicle energy consumption [17]. As the advantages of the intelligent transportation system have been well known to the public, speeding up the improvement of the system has been included in the top priority of the current research in the field of transportation, and it has become the trend of this era [18]. But after all, the intelligent transportation system is a discipline created by the convergence of electronic information technology, Internet of Things technology, automatic control theory, communication technology, and traditional traffic engineering theory, which means that its establishment must rely on a wealth of theoretical foundations $[19,20]$. Then, a lot of infrastructure construction is added to form a large-scale and comprehensive transportation system, so that it can give full play to its role [21].

In order to better alleviate the pressure of urban traffic, this paper conducts research on intelligent video surveillance technology in intelligent traffic. Among them, Chan made a detailed introduction to intelligent video surveillance and believed that the application of dynamic target detection methods is of great significance [22, 23]. Khatri proposed in his paper the application of intelligent transportation systems in contemporary transportation systems and explained the characteristics of smart video surveillance technology and its main development prospects [24]. Cain et al. have a solution for smart video surveillance technology, detailed the feasibility of establishing an intelligent transportation system in the article, applied it to actual engineering, and obtained a large amount of data analysis [25]. Song et al. put forward some common problems in the technology required for system building and proposed the problems that may be encountered in building a corresponding intelligent transportation system [26]. Song et al. proposed a rough classification of dynamic target detection methods in the system and proposed the application of intelligent traffic video monitoring methods in real life and the solution of common problems [27]. Kumar et al. proposed the advantages and disadvantages of intelligent video monitoring technology, which is of great significance to fully solve the urban traffic pressure and believed that this is of great significance for building a harmonious society [28, 29].

To put it simply, this article takes intelligent traffic intelligent video monitoring technology as the main research content, that is, understanding and analyzing the causes and sources of urban traffic pressure, to identify the main directions that need to be resolved, and then to study intelligent traffic intelligent video monitoring technology. Specifically, the main research content of this article is roughly divided into five parts: the first part is the introduction part, which aims to systematically review the main research content of this article from the research background, research purpose, research ideas, and methods; the second part is the theoretical basis, a detailed and systematic summary of the current theories and methods of urban transportation and the current research status of intelligent transportation systems, and it also introduces the current level of convergence of technologies such as the Internet of Things and communication technologies. The third part is related research, which starts with the research of intelligent traffic intelligent video monitoring technology from the aspects of overall and function through specific survey data and detection results. The fourth part is to make a clear summary of the advantages and disadvantages of intelligent video monitoring technology by comparing the effectiveness of intelligent video monitoring technology in real life. The fifth part is the summary and suggestion part of this article. It is a summary of the results of this article and a prospect for the widespread application of intelligent video monitoring technology in intelligent transportation systems.

\section{Proposed Method}

2.1. Intelligent Video Detection Technology. In order to study and realize video tracking in intelligent traffic, objects (vehicles or people) moving in traffic must be determined first, which requires the use of target detection technology. Object detection technology is different from static image analysis but has some similarities [30]. The detection of the target is to divide the picture into the motion interval and the static interval, then analyze the continuous image transformation, extract the structural features of the moving target, and analyze and judge the state of the dynamic target. The image analysis of the moving target is to consider the sequence of the moving image to make up for the feature that the single frame image cannot detect the dynamic target. The commonly used dynamic target detection methods include 
optical flow method, interframe difference method, background difference method, morphology, and so on. The interface difference method is mainly to shoot video segmentation, each frame of the video segmentation, the adjacent two or a few frames in video sequences do bad, and the difference image threshold; the pixel gray level change is greater than the threshold value of points awarded for future, pixel gray sentenced to less than the threshold as the background, so as to detect the target contour [31]. The method has the following formula:

$$
D_{k}(x, y)=f_{k}(x, y)-f_{k-1}(x, y) .
$$

The difference image is obtained by the pixel gray level of the position $f_{(k)}$ of frame $k$ and the position $f_{(k-1)}$ of frame kitchen; threshold judgment is made on the difference image to distinguish foreground and background points, and banalization image is obtained, namely:

$$
\begin{aligned}
& R_{k}(x, y)=0, \\
& D_{k}(x, y)<T, \\
& R_{k}(x, y)=1, \\
& D_{k}(x, y)>T .
\end{aligned}
$$

Compared with other methods of target detection, the principle of interface difference method and operation is very simple, able to quickly detect the moving targets and to the actual monitoring scene has good adaptability, but in practical application, it is difficult to determine the definition of a threshold; the threshold is too small; it is hard to ignore the reality environment such as wind, instruments, and equipment, etc. The threshold is too large. It is easy to the omission of moving target detection and the image cannot detect the complete movement [32]. Therefore, this method is only applicable to the case where the camera is fixed and the environment is less affected.

The main idea of the background subtraction method is as follows: first, select a background image that does not contain the target and then make the difference between the current frame and the background image so as to obtain the target region in the current frame and achieve the goal of target detection [33]. The difference image is obtained by making a difference between frame $k$ image $f_{(k)}$ and background image $B$ :

$$
D_{k}(x, y)=f_{k}(x, y)-B(x, y) .
$$

The selection of background image is very important for the background subtraction method [34]. In order to improve the reliability and accuracy of the background subtraction method, the background should timely select the appropriate background image according to the changes in the environment. In practice, it is very difficult to obtain an accurate objective background due to the influence of light changes, the movement of objects in the background, the changes of background itself, and the noise of image sensor equipment [35].

The concept of optical flow method was proposed by Gibson and Wallach in 1950. When the target in the real world of three-dimensional movement occurs, its corresponding projection point in the two-dimensional plane will change as an image of a light flow refers to the instantaneous velocity in grayscale mode. Based on the optical flow field of each pixel, movements can deduce the target movement in reality. The optical flow field contains the motion information of the actual three-dimensional world target [36]. The optical flow method is mainly used to detect the moving target according to the optical flow property of the moving target with the change of the pixel intensity in the video image sequence [37].

Assuming the pixel $p(x, y)$ in the image, the gray value at time $t$ is $I(x, y, t)$; after a very short time interval $\Delta t$, we can consider that the intensity values of the two remain unchanged, as shown in the following formula:

$$
I(x, y, t)=I(x+\Delta x, y+\Delta y, t+\Delta t) .
$$

Assuming that the change trend of gray value $I$ is smooth, it can be obtained as follows:

$$
I(x, y, t)+\frac{\partial I}{\partial x} \Delta x+\frac{\partial I}{\partial y} \Delta y+\frac{\partial I}{\partial t} \Delta t+e=I(x, y, t) .
$$

$\mathrm{E}$ is the higher order term. Its value is infinitesimal. Dividing both sides by $\Delta t$, the following can be obtained:

$$
\frac{\partial I}{\partial x} \Delta x+\frac{\partial I}{\partial y} \Delta y+\frac{\partial I}{\partial t} \Delta t=0 .
$$

Let $u$ and $v$ represent the velocity vectors in the $X$ and $Y$ directions, respectively, and represent the optical flow of $I$ :

$$
I_{x} u+I_{y} v=-I_{t} .
$$

This is the optical flow constraint equation. According to the global method proposed by Horn and Shuck, a global smoothing constraint is introduced. That is,

$$
e_{c}=\iint\left(\frac{\partial p}{\partial x} u+\frac{\partial p}{\partial y}+\frac{\partial p}{\partial t}\right)^{2} \mathrm{~d} x \mathrm{~d} y .
$$

The optical flow error of the grayscale conservation hypothesis is as follows:

$$
e_{b}=I_{x} u+I_{y} v+I_{t} .
$$

To sum up, the optical flow method can detect moving objects, but the optical flow method is difficult to carry out in real time due to its large and complicated calculation. In addition, the constraint equation of optical flow cannot be satisfied under the condition that the object under test is occluded, multilight source, and multinoise.

With the rapid development of computer technology, we no longer rely on our own eyes for image acquisition but use visual computer-aided systems to process big data information [38]. The important means of the visual aid system is to rely on imaging technology. The information control and processing of the system are realized by the computer. These tasks and contents include the collection, transmission, storage, and processing of visual information. The application goal of the visual computer system in an intelligent 
traffic system is to realize computer-aided traffic guidance. The system has the function of relying on visual sensitive feedback information to realize some visual monitoring task [39]. Computer-aided vision software is composed of automatic control and spatial positioning system [40]. Through the target monitoring technology and computer vision systems, intelligent video monitoring technology for a dynamic target within the scope of visual image analysis, the video image filtering prevents interference light change and natural phenomenon; if the existence of a dynamic target is sensed, the system will immediately upload the dynamic target's license plate number, vehicle direction information, color information, moving speed, and other information to the monitoring and dispatching system for further processing.

\subsection{Evaluation Index of Intelligent Video Monitoring} Technology. The evaluation index can reflect the reliability and feasibility of a system. Different evaluation indexes represent different contents of dynamic target detection methods. The rationality of evaluation indexes is directly related to the authenticity of the final evaluation results. The selection of evaluation indicators should follow the principles of typicality, independence, hierarchy, reliability, and quantifiable.

(1) Typicality: the evaluation indicators should be clear and able to measure and reflect the characteristics of a certain aspect of the system.

(2) Independence: the dynamic target information reflected by different evaluation indicators should be different. If the evaluation indicators are compatible with each other, it should be considered to combine the indicators with the same meaning. "the evaluation of one indicator should not be affected by other indicators.

(3) Hierarchy: the evaluation of the dynamic target detection method is divided into several levels for consideration, and the lower evaluation indexes are reasonably determined according to specific diagnostic indexes to form a structural system.

(4) Reliability: the evaluation index must be able to truly reflect the status of the actual parameters of the dynamic target, and the dynamic target state identified by each evaluation index should be consistent with the actual situation.

(5) Quantifiable: in order to carry out objective analysis and evaluation of targets, quantitative methods must be adopted. Therefore, evaluation indicators should be those that can be quantified according to a certain principle. Dynamic target detection is a complicated system problem. The influencing factors of dynamic target detection are numerous, but when choosing the evaluation index is not possible to all factors into consideration, only in combination with the actual situation to choose the most telling indicator, the traffic problem is the most difficult in the dynamic process of detection of target recognition because the limitation of the current knowledge of the process is not very understanding; that is, the detection of dynamic targets is in a situation where there is limited understanding. Obviously, the fact detection data is the most intuitive information reflecting the parameters of moving targets. Therefore, from the perspective of typicality, reliability, and quantification, it is necessary to use a variety of dynamic target detection methods to test the current difficult dynamic target detection problems to obtain a comprehensive evaluation.

\section{Experiments}

3.1. Related Processing of Experimental Data. In the process of the experiment, a large number of production management data need to be processed, and these data will inevitably have errors. It is also very important to deal with these errors properly. Error processing and analysis of the original data are quite necessary. Otherwise it will affect the positive and negative analysis of the experimental data. Systematic error, random error, and gross error are the main forms of experimental data error. Among them, the errors caused by random factors are called random errors, which are characterized by irregular signs and absolute values but are normally distributed with the increasing number of experiments. Because in the statistical data, due to artificial carelessness, or changes in environmental conditions, instrument instability, and other factors, resulting in the observation error, do not conform to a certain rule of statistical distribution error which is called the measurement error. The systematic error is caused by the reason of measuring instrument, the change of measuring datum, and the influence of external conditions. At present, the test of systematic errors of observed values generally constitutes the corresponding statistics according to the statistical characteristics of observed values, and then the test hypothesis is made according to the probability distribution characteristics, and the discrimination is made by comparing the actual calculated values with the fractional values' test method, variance test method, t-test method, and so on are commonly used measurement methods. To get the data, the first thing to do is to remove the influence of gross error and systematic error and ensure the accuracy of the next step [41].

At home and abroad, the least square method is mainly used to process the monitoring data [42]. The least square method assumes that the observed value contains only accidental error, which is impossible in practice. Therefore, a new theory of systematic error and gross error is generated. At present, the more effective method to deal with the system error is the additional parameter method. There are two methods to deal with a gross error. One is the data detection method which still belongs to the category of least square method. The other is the robust estimation method or the robust estimation method which is different from the least square method. In addition, in the actual monitoring engineering, the measured body is in constant change, and the measuring system is also in motion. Therefore, it can be 
considered that security detection belongs to dynamic measurement, and the error contained in the detection data is necessarily dynamic and time-varying. The gross error in the detection data often makes the statistical distribution difficult to determine or even beyond the general statistical law, and the detection data at different stages are also quite different, so it is difficult to meet the requirements of data processing with traditional methods. Modern error theory holds that the measured truth value cannot be measured, and the existence of quantum effect excludes the existence of a unique truth value, so the error cannot be obtained accurately. The error used in past measurements is actually a deviation. The measured error is actually the uncertainty of the measurement. Uncertainty refers to the degree of uncertainty to the measured value due to the existence of measurement error, and it is the evaluation of the range of magnitude that represents the measured truth value. Considering the characteristics of dynamic target continuity, the reliability of measured value can be considered according to the continuity, progress, and rationality of measured value, that is, the degree of certainty of measured value. High reliability means low uncertainty of measurement value. Low reliability means high uncertainty of measurement. The theory of ascertain mathematics can be used to describe the reliability of data. The following is the method of ascertain mathematics for the analysis and error processing of original data.

\subsection{The Establishment of the Experimental Model of Intelligent} Video Monitoring. It is planned to build a video image sharpening prototype system based on the guidance of moving object detection, which includes the following functions: video file reading, moving object detection and extraction, moving object sharpening processing, and video file saving and display. Moreover, the results of the video image sharpening under the guidance of different motion signs are compared. Through this algorithm, traffic video can be analyzed, and moving objects in the video can be clarified, providing a basis and support for the research and development of an intelligent traffic monitoring system.

3.2.1. The System Implements the Hardware Device. PC: the $\mathrm{CPU}$ is Intel 4 , the memory size is $4 \mathrm{G}$, the hard disk size is 240G. One digital camera: it is used to shoot video files of moving vehicles in intelligent transportation [41]. The image size is 320 times 240 , and the acquisition rate is 15 frames per second.

3.2.2. System Design. The module structure of the system is mainly composed of a video reading module, moving object detection module, clearness processing module, and video display module. Video reading module is mainly responsible for storing the video images input by video input devices such as cameras into the computer cache frame by frame, providing the system with a frame-by-frame image sequence for further processing. This system can process real-time video input from the camera. The moving object detection module uses the method of interface difference and optical flow to detect the moving object, then performs morphological filtering on the detected foreground binary image, marks the connected region, and finally extracts the moving object, which provides the basis for the video image sharpness processing [43]. The deburring processing module firstly deburrs the image, then clarifies the extracted moving target area, and adopts the method of specific area enhancement. In order to display the result of the final clarification processing, the system displays the results of video images in the form of RGB. In the case of the same degree of blurriness of the video image, the constrained least squares algorithm is better than the Wiener filter to restore the clearing effect, and as the noise increases, the image becomes blurred, and the clarity of the image recovered is worse. Therefore, the recovery effect is worse, so the constrained least squares algorithm is selected to remove the motion blur, and the specific image quality evaluation under different noises is shown in Table 1.

Table 1 data represent the image quality evaluation table under different noises. The target position signal obtained from the intelligent traffic scene by video analysis technology contains various noises. The filtering algorithm of thought is in the sequence by predicting, update two steps iterative update constantly, causing the error of the system noise and observation noise to decrease gradually in order to obtain the best state parameter, achieve the goal of eliminating noise, and effectively avoid the traditional image of the video data after due to noise operator to bring further the defect of fuzzy. Wiener filter algorithm and constrained least squares method are used finally to remove the motion blur with the constrained least squares method, using the fuzzy kalian filter to eliminate noise and filtering based on a specific area to enhance the motivation method; this method can be a more outstanding image of some important information, make the image clearer, can reduce the amount of calculation, and at the same time improve the real-time performance.

\section{Discussion}

4.1. Analysis of Intelligent Video Monitoring Technology. There are many reasons for unclear video images in traffic monitoring, and different methods are adopted for different reasons. For the specific application of intelligent traffic monitoring, the main considerations are motion blur, noise blur, and low contrast blur [44]. In view of this, wiener filtering and constrained least square algorithms are, respectively, used for restoration and clearness processing. The variation trend of the effect is shown in Table 2:

It can be seen from the data in Table 2 that the effect is best when the gain value is 0.5 . However, when the gain value is greater than 0.5 , the predicted value of pixel will be higher than the actual value, so the effect of processing will be decreased.

The system carries out experiments on a number of traffic road video files collected, deburring the relatively fuzzy video, and then sharpening the video images of specific areas based on the detection guidance. In order to evaluate 
TABLE 1: Image quality evaluation table under different noises.

\begin{tabular}{lccc}
\hline & MAE & MSE & SNR \\
\hline ó=2 & 47921 & 67498 & 19.645 \\
Ó=4 & 87042 & 85079 & 10.297 \\
\hline
\end{tabular}

TABLE 2: Image quality evaluation table.

\begin{tabular}{lcc}
\hline Gain value & $\Delta R$ & NMSE \\
\hline Gains $=0.25$ & 0.432 & 0.004034 \\
Gains $=0.50$ & 0.259 & 0.002534 \\
Gains $=0.75$ & 0.542 & 0.005132 \\
\hline
\end{tabular}

the quality and degree of image sharpening after the system sharpening processing, this paper compares the images before the sharpening processing with the processed images and compares the improved detection algorithm in this paper with the images processed by other common detection methods, so as to highlight that the improved algorithm is better. In this paper, the average absolute error, mean square error, normalized homogenization error, SNR, and minimum correlation number error are used to evaluate the image quality, as shown in Figure 1:

To read in the traffic video file, read the image, respectively, using frame differential method and background subtraction method, optical flow method, and frame flow method to detect moving targets, and then to detect the binary motion area of the postprocessing, such as median filter and morphological filtering and extracting the movement area, and then to extract the motion area of the recovery process, based on regional enhanced image clearer processing, finally display video images. As can be seen from the data in Figure 1, in several image motivation based on the detection algorithm, the MAE, MSE, NMSE, and $\Delta R$ values of the improved algorithm proposed in this paper are smaller than those of other algorithms, and the resulting value is larger than that of other algorithms, which explains the processed image is clearer and the difference between the original image is very small; this means that the improved algorithm in video image processing effect is better than other algorithms. After the application of intelligent video monitoring technology, compared with the traditional traffic system, the images are more specific and clearer, more information is obtained from the images, and the feedback is faster. The data measurement time has been shortened by one hour, the aggregation time has been changed from three hours to two hours, and the analysis time has been shortened by half. In these fastpaced times, every second can be the difference between success and failure. With the application of intelligent video monitoring system, urban residents can get feedback more quickly when they travel, so as to adjust their travel routes to reduce the unnecessary time wasted in traffic jams, which also extends the time available to residents in a hidden way and provides a guarantee for meeting residents' other needs. For the transportation department, a large amount of redundant data processing time can be reduced, so that manpower and material resources can be arranged more rationally.

Traffic flow monitoring is an important component of intelligent traffic. To study the function of video tracking technology in intelligent traffic, it is necessary to realize the function of traffic flow monitoring. Designated in the video picture need to monitor the alert area when moving inside the vehicle; based on the intelligent video surveillance technology, analysis platform immediately sends alarm information to the terminal interface and the vehicle's trajectory according to target motion trajectory in the management of terminal monitoring screen on the basis of the realization of target detection; the detected vehicle enters can automatically detect the video traffic detection scope and can be the flow in the management of terminal display; observation data is shown in Figure 2:

As can be seen from Figure 2, the real-time traffic flow of the road can be clearly understood through intelligent video monitoring technology. Traffic flow in parking lots, highways, central roads, and suburban intersections was observed. This means that if there is a traffic jam at the central intersection during the rush hour, the intelligent video monitoring technology can be used to give timely feedback so that the majority of residents can stagger the traffic flow.

4.2. Analysis of Dynamic Target Detection Method. The basic principle of dynamic object detection is to input the image of a dynamic object through a video camera and complete the digitization. In the process of image acquisition, some noise signals that affect image quality are generated, so before processing these digital images, digital image filtering should be carried out first. For the preprocessed images, corresponding video detection procedures can be conducted to extract the specific parameters of dynamic targets. Robustness, accuracy, and processing speed are the main indexes in the performance analysis of a moving date detection algorithm. The robustness of the algorithm means that the algorithm can work automatically and continuously and is less sensitive to the influence of noise, light, and weather factors. The processing speed of the algorithm is the realtime performance of the algorithm, as shown in Table 3:

It can be seen from Table 3 that the traditional dynamic target detection method has a long cycle and can no longer 


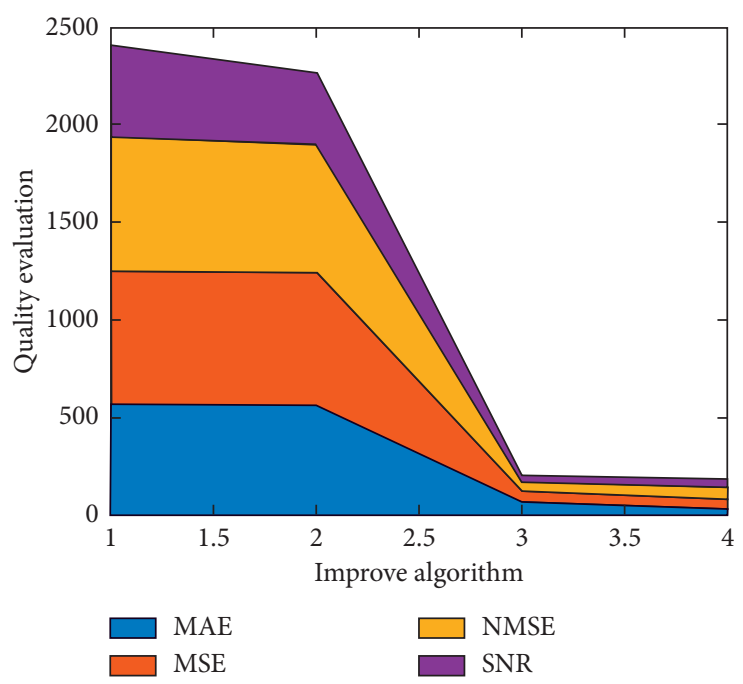

Figure 1: Quality evaluation of the improved algorithm.

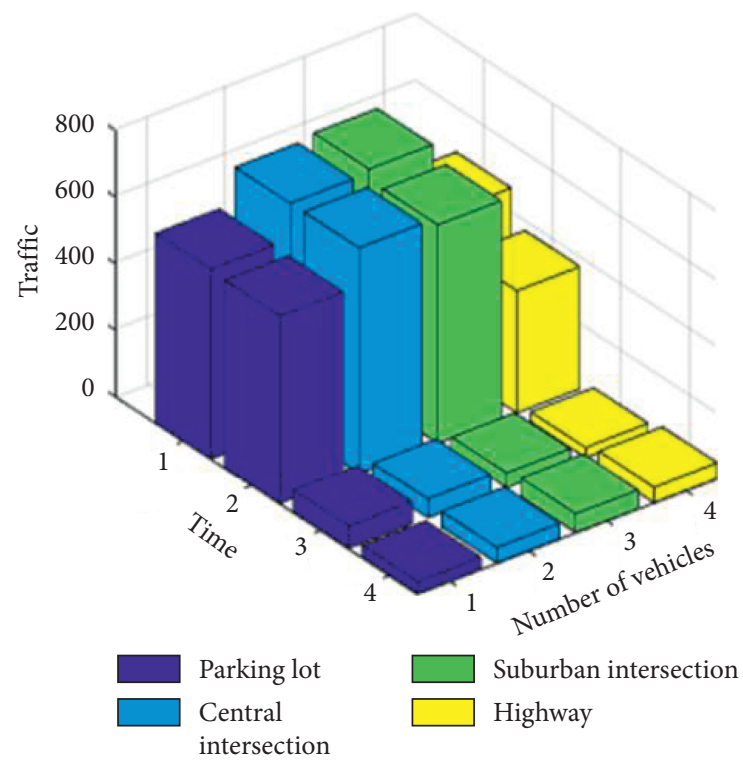

Figure 2: Traffic monitoring.

adapt to the current fast-paced era. It can be seen that the improved detection algorithm has good performance in robustness, accuracy, and processing speed. In the practical application, people often use the improved algorithm or a combination of several algorithms; then the performance will be different. Therefore, in the selection of algorithms, we should try to overcome the shortcomings of each algorithm in view of its advantages and disadvantages and give play to their respective advantages to improve the detection effect. Time is critical for dynamic target detection in intelligent transportation systems. So the development prospect of the improved algorithm is very good.

The improved algorithm integrates the multifeature extraction method to extract and analyze the dynamic target and integrates the classical algorithm to realize the dynamic target tracking of multifeature adaptive parameters, and proposes the traffic flow detection framework and process of dynamic target information, and expounds the traffic flow detection method based on video images. However, the field of transportation involves not only computer vision recognition, pattern recognition, and other information domain expertise but also a large part of factors that depend on the implementation of traffic rules of human factors and the natural environment. Therefore, in view of this concern, the dynamic target detection method is tested in a comprehensive environment to verify the correctness of the dynamic target. See Figure 3 for details.

As can be seen from Figure 3, the correctness test of the improved algorithm is much higher than that of the traditional method, which indicates that the improved algorithm has great potential. However, due to the limitation of the natural environment and the influence of human factors, the accuracy of the improved algorithm is not stable enough, which has some limitations on its application. If the improved algorithm is to be further introduced into the traffic field, its stability needs to be improved. If this problem can be completely solved, the pressure of urban traffic will be greatly relieved.

Video image sharpening processing is the core technology of all kinds of intelligent video monitoring systems. It is the cornerstone of various subsequent high-level processing and applications, such as target tracking, behavior recognition, target classification, and behavior analysis. To explore the ability to calmly cope with various changes in the complex environment, and accurately, quickly, and stably detect moving objects. According to relevant standards, the safety factor index standards are established, as shown in Figure 4:

As can be seen from Figure 4, the improved algorithm has the highest pixel and the best definition. The frame difference method has high robustness but slow accuracy and processing speed. Background differential flu robustness is low; accuracy and processing speed are fast. The robustness and processing speed of the optical flow method are slow and the accuracy is high. 
TABLE 3: Algorithm performance comparison.

\begin{tabular}{lccc}
\hline Detection algorithm & Frame difference method & Optical flow method & Improve algorithm \\
\hline Robustness & High & Low & High \\
Accuracy & Low & Higher & High \\
Processing speed & Fast & Slow & General \\
\hline
\end{tabular}

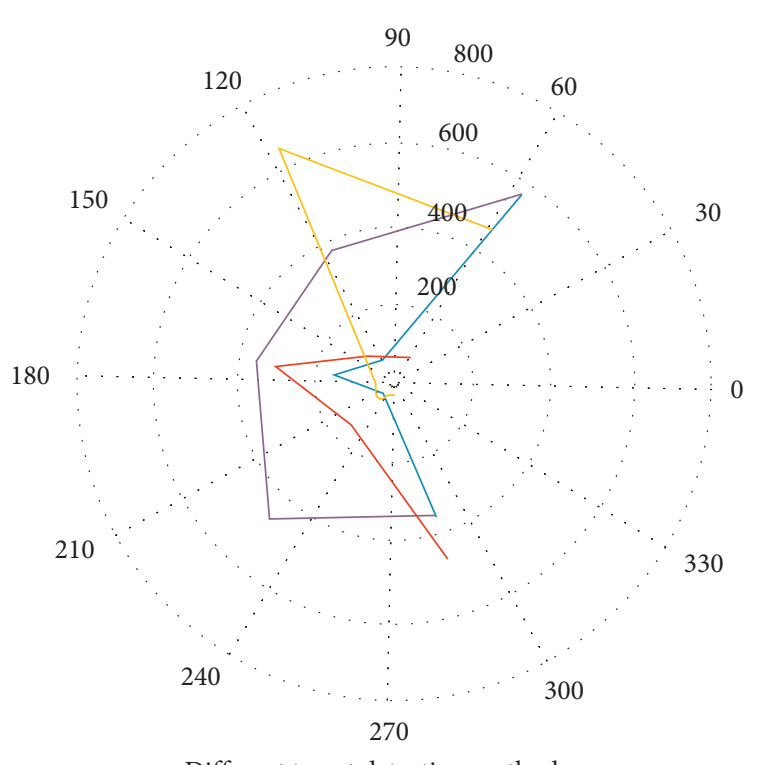

Different target detection methods

— Background method _ Improve algorithm _ Interframe method Optical flow method

FiguRE 3: The correctness of different methods.

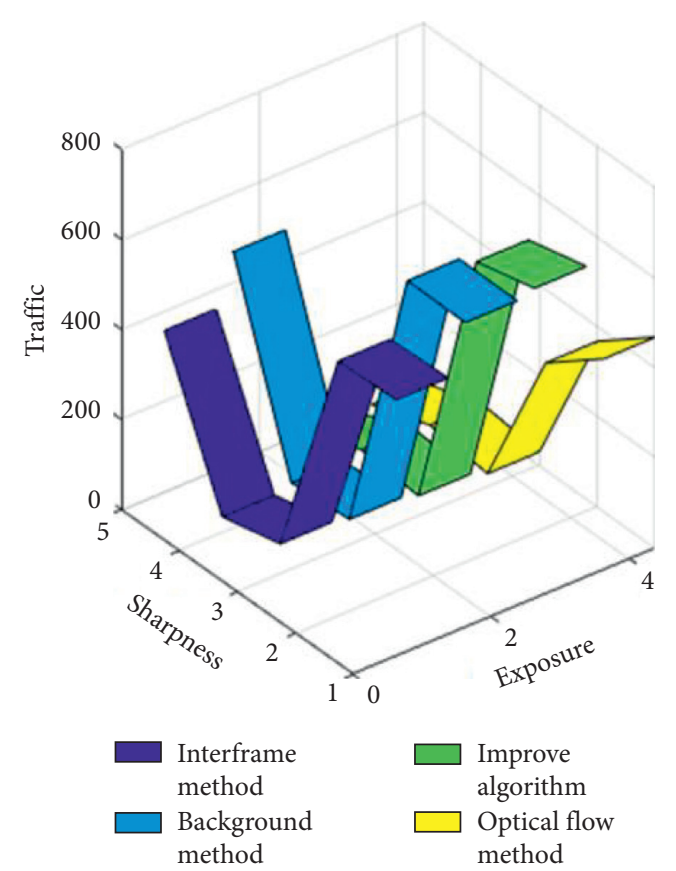

Figure 4: Pixel size comparison.

\section{Conclusions}

(1) This article analyzes the situation that with economic development, more and more residents are driving by car, which poses a great challenge to urban transportation to a certain extent. The main problem is that the speed of increasing vehicles is not consistent with the speed of road construction, resulting in the present problems such as traffic jams and long travel times in urban transportation which further affect the residents' life experience and are not conducive to the long-term development of the city. In the face of this increasingly severe traffic situation, the emergence of intelligent video monitoring systems provides a direction for solving such problems. Therefore, this article conducts research on intelligent video monitoring systems in order to deepen the understanding of emerging technologies and better apply them to go in real life.

(2) This article introduces the global positioning system, communication technology, and traditional transportation field theoretical technology. Based on the theory, it is clear that video surveillance technology is the product of multidisciplinary convergence and clarifies the definition and related characteristics of video surveillance technology. The classic moving target detection method is briefly introduced, and an improved target detection algorithm of the interframe difference optical flow method is proposed. It maintains the advantages of the fast and efficient extraction of moving targets by the interframe difference method. Moreover, the advantages of reliable optical target extraction and strong antinoise ability of optical flow method are maintained. The moving target detection method based on this can effectively extract the traffic target area from the surveillance video at the intersection.

(3) This paper made a certain analysis of the performance of the intelligent video monitoring technology and compared the improved algorithm with the interframe difference method, the background light method, and the optical flow method. X. It has improved by 20 percentage points in accuracy and has advantages unmatched by other target detection methods. It not only saves a lot of manpower and resources but also greatly improves efficiency. This study of intelligent video surveillance technology in intelligent traffic can effectively increase the awareness of intelligent video surveillance technology, 
improve the level of intelligent video surveillance technology, and shorten the data measurement time by one hour and the aggregate time from three hours to two hours The reduction of analysis time is even more obvious, and the time spent is reduced by half. Relieving the pressure on urban traffic roads has also greatly reduced the incidence of traffic accidents, which is conducive to the construction of a harmonious socialist society.

\section{Data Availability}

All the data in this article are real and available.

\section{Conflicts of Interest}

There are no potential conflicts of interest in our paper. All authors have seen the manuscript and approved to submit it to the journal. The authors confirm that the content of the manuscript has not been published or submitted for publication elsewhere.

\section{Acknowledgments}

This work was supported by Chongqing Big Data Engineering Laboratory for Children, Chongqing Electronics Engineering Technology Research Center for Interactive Learning, Project of Science and Technology Research Program of Chongqing Education Commission of China (No. KJZDK201801601).

\section{References}

[1] T. Gaber, S. Abdelwahab, M. Elhoseny, and A. E. Hassanien, "Trust-based secure clustering in WSN-based intelligent transportation systems," Computer Networks, vol. 146, pp. 151-158, 2018.

[2] Z. Lv, S. Zhang, and W. Xiu, "Solving the security problem of intelligent transportation system with deep learning," IEEE Transactions on Intelligent Transportation Systems, pp. 1-10, 2020.

[3] X.-H. Zhang, S. Zhang, S. Fang, and X. Cao, "Clearing research on fog and dust images in coalmine intelligent video surveillance," Journal of the China Coal Society, vol. 9, no. 4, pp. 32-33, 2016.

[4] J. M. Huang, "Research on internet of vehicles and its application in intelligent transportation," Applied Mechanics \& Materials, vol. 321-324, pp. 2818-2821, 2013.

[5] C.-H. Chen, "An arrival time prediction method for bus system," IEEE Internet of Things Journal, vol. 5, no. 5, pp. 4231-4232, 2018.

[6] L. Jinn, H. Goo, M. Liu, and J. Xiao, "Video event detection technology and its application in intelligent transportation," Applied Mechanics \& Materials, vol. 20, no. 9, pp. 1-2, 2017.

[7] S. Wan, X. Li, Y. Xue, W. Lin, and X. Xu, "Efficient computation offloading for Internet of vehicles in edge computing-assisted 5G networks," The Journal of Supercomputing, vol. 76, no. 4, pp. 2518-2547, 2020.

[8] R.-H. Zhang, F. You, F. Chen, and W. Q. He, "Vehicle detection method for intelligent vehicle at night time based on video and laser information," International Journal of Pattern
Recognition \& Artificial Intelligence, vol. 32, no. 4, Article ID 1850009, 2018.

[9] C. Jinn, W. Dan, and K. Cain, "Research and implementation of image compression in intelligent video surveillance system based on SOPC," IEEE Sensors Journal, vol. 21, no. 1, pp. 10-20, 2016.

[10] J. Liu, H.-Q. Zhang, and Y. Liu, "Research on optimal selection of moving target defense policy based on dynamic game with incomplete information," Act Electronica Sonica, vol. 21, no. 15, pp. 110-120, 2018.

[11] Z. Lv, W. Kong, X. Zhang, D. Jiang, H. Lv, and X. Lu, "Intelligent security planning for regional distributed energy internet," IEEE Transactions on Industrial Informatics, vol. 16, no. 5, pp. 3540-3547, 2020.

[12] B. Cui, J. Cui, and D. Yong, "Intelligent security video surveillance system based on deviance technology," Journal of Mechanical Strength, vol. 42, no. 1, pp. 1-12, 2016.

[13] M.-G. Kim, H.-M. Moon, and Chung, "A survey and proposed framework on the soft biometrics technique for human identification in intelligent video surveillance system," Journal of Biomedicine \& Biotechnology, vol. 42, no. 35, pp. 5-6, 2018.

[14] R. Parada, J. Melià-Seguí, and R. Pous, “Anomaly detection using RFID-based information management in an IoT context," Journal of Organizational and End User Computing, vol. 30, no. 3, pp. 1-23, 2018.

[15] N. Li, T. Zhao, and H. Jiang, "Research on dynamic coupling characteristic of rootless under actuated redundant robots," Journal of Mechanical Engineering, vol. 12, no. 21, pp. 12-16, 2016.

[16] W. Song, Y. Yang, M. Fu, Y. Li, and M. Yang, "Lane detection and classification for forward collision warning system based on stereo vision," IEEE Sensors Journal, vol. 18, no. 12, pp. 15-16, 2018.

[17] T. Yu, B. L. Hu, and Gao, "Research on dynamic tracking and compensation method for hyperspectral interference imaging," Gangs Xebio/Act Photonic Sonica, vol. 45, no. 7, pp. 25-36, 2016.

[18] I. Butun, P. Österberg, and H. Song, "Security of the Internet of things: vulnerabilities, attacks, and countermeasures," IEEE Communications Surveys \& Tutorials, vol. 22, no. 1, pp. 616-644, 2020.

[19] Y. Zhang, H. Liu, J. Wu, J. He, and C. Zhang, "Statistical analysis for performance of detection and imaging of dynamic targets using the geostationary interferometric microwave sounder," IEEE Journal of Selected Topics in Applied Earth Observations \& Remote Sensing, vol. 1, no. 1, pp. 3-11, 2018.

[20] Z. Lv and A. Kumar Singh, "Big data analysis of Internet of things system," ACM Transactions on Internet Technology (TOIT), 2020.

[21] Y.-T. Chan, S.-J. Wang, and C.-H. Tsai, "Extracting foreground ensemble features to detect abnormal crowd behavior in intelligent video-surveillance systems," Journal of Electronic Imaging, vol. 64, no. 17, pp. 12-15, 2015.

[22] J. Lan, S. Li, and S. Wu, "Research on ensemble classification model of Trojan traffic detection," Journal of Xian Tongan University, vol. 49, no. 8, pp. 4-9, 2015.

[23] M. Elhoseny, "Multi-object detection and tracking (MODT) machine learning model for real-time video surveillance systems," Circuits, Systems, and Signal Processing, vol. 39, no. 2, pp. 611-630, 2019.

[24] V. Khatri, "Intelligent video surveillance using soft biometrics," Xitsonga Lila You Shavian/System Engineering Theory and Practice, vol. 35, no. 5, pp. 12-13, 2015. 
[25] L. Cain, J. Xu, and L. Jinn, "Research on agricultural surveillance video of intelligent tracking," Journal of Mechanical Engineering, vol. 52, no. 4, pp. 13-14, 2016.

[26] G.-C. Song, Y.-X. Na, F.-Y. Yang, and S.-H. Cao, "Research on portable intelligent monitoring system based on video server," Journal of Intelligent \& Robotic Systems, vol. 92, no. 3, pp. 1-12, 2017.

[27] W. Song, J. Zhang, W. Zhang, Y. Wang, Q. Zhuo, and W. Shi, "Research of intelligent video surveillance in substation based on ViBe," Chinese Journal of Scientific Instrument, vol. 99, no. 2, pp. 27-28, 2016.

[28] N. Kumar, J.-H. Lee, and J. Rodrigues, "Intelligent mobile video surveillance system as a bayesian coalition game in vehicular sensor networks: learning automata approach," IEEE Transactions on Intelligent Transportation Systems, vol. 39, no. 6, pp. 11-12, 2016.

[29] H. Elhoseny, M. Elhoseny, A. M. Riad, and A. E. Hassanien, "A framework for big data analysis in smart cities," The International Conference on Advanced Machine Learning Technologies and Applications (AMLTA 2018), vol. 723, pp. 405-414, 2018.

[30] N. Babaguchi, A. Cavallaro, R. Chellappa, F. Dufaux, and L. Wang, "Special issue on intelligent video surveillance for public security and personal privacy," IEEE Transactions on Information Forensics \& Security, vol. 16, no. 1, pp. 8-15, 2017.

[31] S. Wan, Y. Xia, L. Qi, Y.-H. Yang, and M. Atiquzzaman, "Automated colorization of a grayscale image with seed points propagation," IEEE Transactions on Multimedia, 2020.

[32] Z. You, G. Chen, and B. Wang, "Intelligent control based on a-support vector regression theory for regional traffic signal system," Journal of Computer Applications, vol. 37, no. 2, pp. 34-36, 2015.

[33] M. Chippewa, M. Trashy, and T. Moroccan, "Data fusion in a mufti agent system for person detection and tracking in an intelligent room," Studies in Computational Intelligence, vol. 57, no. 5, pp. 12-15, 2015.

[34] I. B. Guo, "Target detection method based on beam forming output DC response of coinsurance matrix," Journal of Computer Applications, vol. 45, no. 5, pp. 11-16, 2017.

[35] Y. Zhao and C. Yang, "Information fusion robust guaranteed cost Kalman estimators with uncertain noise variances and missing measurements," International Journal of Systems Science, vol. 50, no. 15, pp. 2853-2869, 2019.

[36] V. Agrawal, B. K. Panigrahi, and P. M. V. Subarea, "Intelligent decision support system for detection and root cause analysis of faults in coal mills," IEEE Transactions on Fuzzy Systems, vol. 32, no. 99, pp. 1-5, 2016.

[37] S. Ding, S. Qu, Y. Xi, and S. Wan, "A long video caption generation algorithm for big video data retrieval," Future Generation Computer Systems, vol. 93, pp. 583-595, 2019.

[38] Z. Li, R. A. Hassan, and Mohammad, "A hierarchical framework for intelligent traffic management in smart cities," IEEE Transactions on Smart Grid, vol. 45, no. 99, pp. 11-23, 2017.

[39] J. Yang, C. Wang, B. Jiang, H. Song, and Q. Meng, "Visual perception enabled industry intelligence: state of the art, challenges and prospects," IEEE Transactions on Industrial Informatics, p. 1, 2020.

[40] A. A. Rahmani Hosseinabadi, J. Vahidi, B. Saemi, A. Kumar Sangaiah, and M. Elhoseny, "Extended genetic algorithm for solving open-shop scheduling problem," Soft Computing, vol. 23, no. 13, pp. 5099-5116, 2019.

[41] M. R. Reddy, K. G. Srinivasa, and B. E. Reddy, "Smart vehicular system based on the Internet of things," Journal of
Organizational and End User Computing, vol. 30, no. 3, pp. 45-62, 2018.

[42] X. Chen, Y. Xu, L. Meng et al., "Non-parametric partial least squares-discriminant analysis model based on sum of ranking difference algorithm for tea grade identification using electronic tongue data," Sensors and Actuators B: Chemical, vol. 311, Article ID 127924, 2020.

[43] H. Song and M. Brandt-Pearce, "A 2-D discrete-time model of physical impairments in wavelength-division multiplexing systems," Journal of Lightwave Technology, vol. 30, no. 5, pp. 713-726, 2012.

[44] B. Wang and L. L. Chen, "New results on fuzzy synchronization for a kind of disturbed memristive chaotic system," Complexity, vol. 2018, Article ID 3079108, 9 pages, 2018. 